\title{
Development and implementation of the Short Term Activity Tracker and Mine Control Trigger Response System
}

\author{
C Chester Newcrest Mining Limited, Australia \\ D Cuello Newcrest Mining Limited, Australia \\ G Basson Institute of Mine Seismology, Australia
}

\begin{abstract}
Seismic risk management at mining operations is predominately reliant on analysis and interpretation by ground control engineers, who must also manage all other mine site geotechnical hazards. Seismic systems run in real time collecting seismic parameters. However, the analysis, interpretation and resultant actions can be intermittent depending on available resources. Stress-induced seismicity during cave initiation and propagation is a constantly evolving hazard that requires a high level of continuous monitoring and examination. The authors have assisted in the development of integrated seismic monitoring and trigger response software applications within the Ticker3D seismic visualiser and analysis application, developed by the Institute of Mine Seismology (IMS). The benefits of the integrated applications include real-time monitoring of key seismic parameters and automatic trigger response, aiming to reduce workforce exposure to hazardous seismic conditions. Using measured ground motion as a monitoring input enables an almost immediate trigger response to a threshold breach via the Mine Control Trigger Response System. The continuity in seismic data interpretation and triggered mitigation controls are also recognised advantages to the applications.
\end{abstract}

Keywords: seismic risk management, TARP, ground motion, Short Term Activity Tracker, seismicity, mitigation controls, exposure

\section{Introduction}

Analysis, interpretation and response to seismic activity is an inherent part of geotechnical engineering for deep and high-stress mines in Australia. Trigger Action Response Plans (TARPs) are often used to coordinate proactive and reactive responses to seismic activity and large seismic events. Although TARPs provide a response framework and trigger levels, it is often still the job of the geotechnical engineer to interrogate the seismic database and determine if a trigger threshold has been breached. This human component results not only in delays in response action initiation, but also inconsistencies in data interpretation and risk categorisation. This reduces the value of TARPs as a risk management tool. Having dealt with seismic risk management over a combined career span of 30 years, the authors, in conjunction with the Institute of Mine Seismology (IMS), developed an integrated monitoring and response software application. The application is designed for automatic tracking of seismic parameters and triggering responses following threshold breaches. The key features of the applications are built upon existing features of the IMS Ticker3D software package, and can be summarised as follows:

- Continuous and real-time monitoring of seismicity and system health.

- Rapid and automatic trigger response initiation.

- Multiple monitoring parameters, event activity rate, event magnitude, peak ground velocity (PGV) and system health.

- Trigger response continuity through reduced human intervention.

- Electronic document management (TARP). 
- Alert confirmation and parameter change logs.

- Trigger threshold impact evaluation.

- Effective communication tool.

- Long-term seismic system monitoring.

\section{Seismic risk management - historic approach}

A qualitative or quantitative seismic risk assessment approach, used to assess the safety risk associated with seismicity has previously been suggested by Heal et al. (2006), whereby:

Seismic Risk $=$ Seismic Hazard $\times$ Excavation Vulnerability $\times$ Workforce Exposure

Any reduction in one of the three factors will result in a direct reduction in seismic risk. The work undertaken and detailed in this publication focuses primarily on reducing workforce exposure in caving operations through the use of automatic software applications for monitoring of the seismic environment and to initiate mitigation controls. Historically, there have been minimal adoptions of automation applied to seismic risk management, with reliance on engineers to review and interpret seismic trends and enact any mitigating controls. This process is often undertaken with the use of TARPs, used to categorise the level of risk and outline mitigating controls.

TARPs are now common within the mining industry covering numerous aspects of both underground and open pit mining. They are a useful communication tool that can be used to classify the risk condition and highlight normal operating conditions, abnormal conditions where a risk may be emerging, or emergency conditions.

Although TARPs are fundamentally reactive in their management of hazards, whereby predefined response actions are triggered as a reactive response to breached thresholds, for the purpose of this paper two classifications, 'proactive' and 'reactive' TARPs have been used. This is to distinguish between TARPs that act upon precursory event triggers (proactive TARPs) and those that are activated post an event's occurrence (reactive TARPs). The aim of proactive TARPs being hazard prevention or reduction in hazard probability and/or hazard exposure. Whereas reactive TARPS, which are more common, deal with the aftermath of a hazard's occurrence. Here, the goal is to reduce further exposure to sympathetic hazards such as aftershocks or delayed falls of ground.

TARPs are generally broken down into a number of levels for the identified hazard, which have set conditions or thresholds that correspond to predetermined actions or mitigation controls. The primary purpose of a TARP is to assist decision-makers implement response actions and minimise delay. Other benefits include the capture of lessons learnt from previous occurrence and mock simulations, and standardised responses to risks. Although the benefits of TARPs for risk management are well recognised, there are identified shortfalls with their implementation and management, which are discussed in relation to seismic TARPs in the following sections.

\subsection{Seismic TARPs}

The TARP framework lends itself to seismic risk management at mining operations, with mining regulatory bodies considering their use as 'good practice'. The effectiveness of the TARP platform and its associated controls are limited by seismic monitoring technology, lack of automation, and the requirement for human intervention. The following section discusses some of the ways in which a TARP can be applied to seismic risk management and the associated shortfalls.

\subsubsection{Proactive seismic TARPs}

One of the more challenging aspects of seismic risk management is the development of an effective proactive seismic TARP, whereby seismic parameter(s) are monitored in a way so as to determine the onset of increased seismic risk and potential for more adverse seismic conditions. Proactive seismic TARPs have threshold triggers aimed at implementing mitigation controls before the large event occurs. One example of 
a proactive seismic risk management approach is to monitor seismic event activity rate to provide an indication of the seismic hazard state. An increase in seismic activity rate is considered by Mendecki (2016a) as an indicator of an elevated seismic hazard state.

However, the application of an event activity rate-based TARP often relies on the subjective interpretation of individual engineers and is typically undertaken intermittently throughout the day and during working hours. As the induced-stress conditions at a caving operation, especially during the early stages of cave undercutting, initiation and propagation, are constantly evolving, the seismic environment and associated hazard also adjust constantly. Under an intermittent review approach, any rapid change in seismic activity outside of a review period has the potential to be overlooked or identified only after a period of increased seismic risk exposure.

\subsubsection{Reactive seismic TARPs}

Reactive seismic TARPs deal with the aftermath of a large seismic event, which includes the potential for aftershocks and other sympathetic hazards such as rockfall. The aim of the TARP is to rapidly and safely reduce personnel exposure to the current hazard state and any cascading hazards, which may have elevated states as a result of the initial event(s). Reactive seismic trigger thresholds are often based on an event's magnitude and proximity to excavations, being major components of seismic hazard definition. There is inherently a delay between event occurrence and response initiation, as events must first be manually processed, either onsite or remotely, to ensure authenticity and accurate event location and magnitude. Once processed and a threshold breach realised, an automated alert can be generated either via an automated voice message, SMS or e-mail. This identified delay leaves the workforce unnecessarily exposed to potential sympathetic hazards such as damaging aftershocks or unstable ground conditions resulting from an initiating event. In addition to the delays associated with event processing, there can be further delays associated with data interpretation and communication of required mitigation controls; again adding to the exposure time of the workforce to a possibly heightened seismic hazard.

The management and implementation of reactive TARPs require good administration and document version controls to ensure all stakeholders have an up-to-date version of the TARP and can implement it when required. Any failure in these administration controls can lead to unnecessary exposure of the workforce.

\section{Short Term Activity Tracker}

In response to the recognised shortfalls detailed above, a Short Term Activity Tracker (STAT) and Mine Control Trigger Response System (MCTRS) have been developed to form an integrated monitoring and alert system that aims to reduce workforce exposure to seismic hazards. The following sections detail the two applications and provide design criteria for their implementation.

The STAT application is built within the IMS suite of seismic analysis and visualisation software applications. It enables the operator to continuously monitor the seismic activity rate and other seismic parameter within predefined monitoring areas or 'polygons'. The application can be coupled with the MCTRS to form an automatic monitoring and alert system, with the overall aim of reducing workforce exposure to seismic hazard. The application was developed with IMS in response to the author's recognition of the shortfalls associated with current seismic risk management systems.

The application has two primary inputs: the monitoring area (polygon), and multiple seismic parameter thresholds (activity rate, event magnitude and ground motion). The system health of the seismic monitoring system can also be monitored in real time to signal any reduction or loss of monitoring capability and subsequent reduction in seismic risk management effectiveness.

One of the key benefits of the use of polygons, which form the basis of the application, is the ability for the geotechnical engineer to select areas according to the level of seismic risk and apply appropriate trigger thresholds. It must be noted that the use of polygons has its shortfalls in that alerts and mitigation controls are applied to the entire polygon volume, while the hazard may occur in only one part. 
Historically, it has been common practice to focus attention on areas of clustered seismicity and associated seismic hazard while overlooking other seismic variables that define the seismic risk, such as workforce exposure and excavation vulnerability. Heal et al. (2010) proposed the use of the rockburst damage potential index, which looked at excavation vulnerability potential among other variables to determine the rockburst hazard state. Although the approach has been integrated into the ACG's mXrap software (Harris \& Wesseloo 2015), the approach is considered a longer term monitoring and analysis tool.

Through the development and implementation of the STAT system at two caving operations, the authors have identified several criteria that should be considered when creating STAT monitoring polygons and determining trigger thresholds. An example of these considerations can be seen in Figure 1, which illustrates the monitoring polygons at a cave mining operation. The criteria are summarised below, while more details are provided in the following sections.

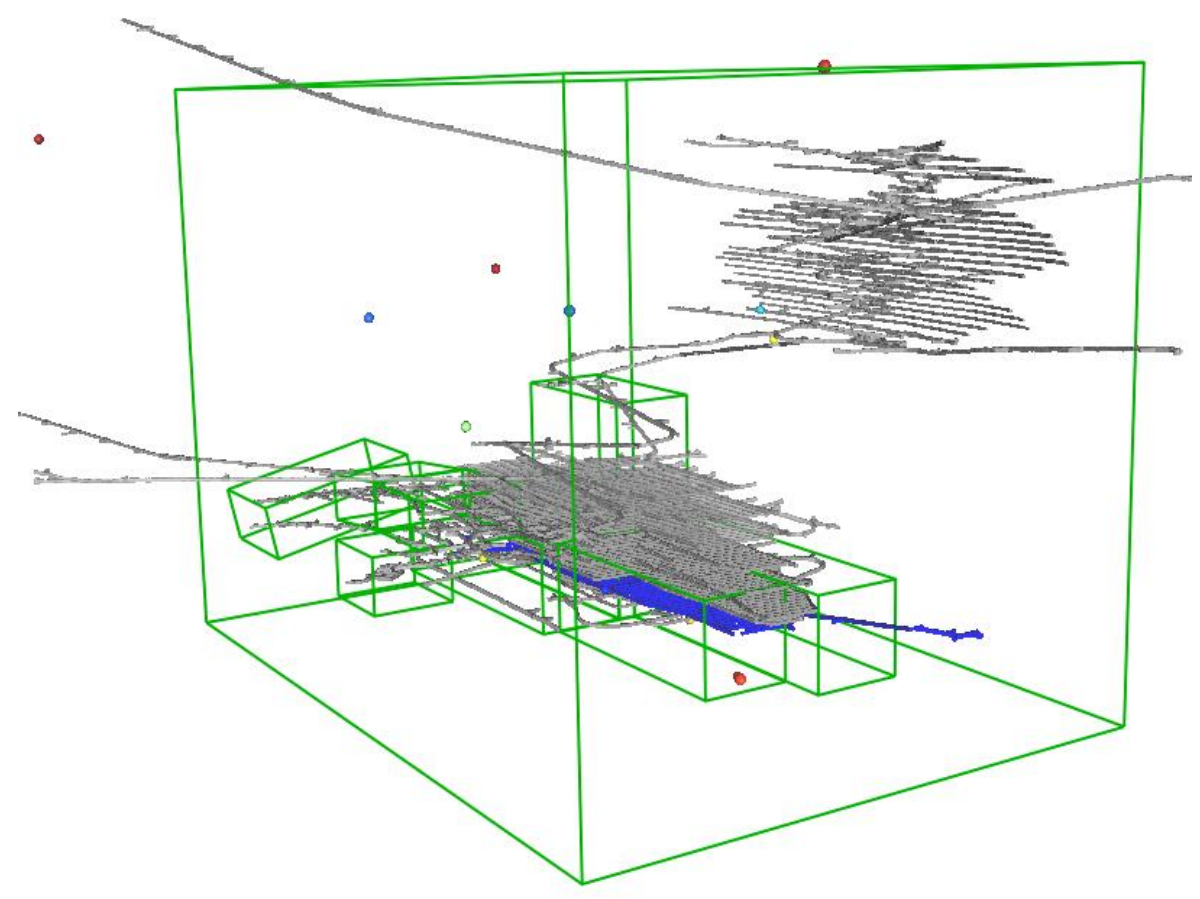

Figure 1 Examples of monitoring polygons at a caving mining operation

Polygon selection criteria:

- Workforce exposure. Areas of high exposure, such as workshops and crusher chambers, where constant exposure of personnel outside of vehicles occurs.

- Infrastructure exposure. Areas of critical infrastructure, both production and safety related (secondary egress).

- Seismic catalogue size. The polygon must contain a sufficient seismic catalogue of events which are considered background and not influenced by blasting or large events.

- Installed seismic monitoring capacity. To achieve the full functionality of the STAT application, monitoring polygons ideally have a minimum of two functioning sensors inside the polygon.

Threshold determination criteria:

- Ground support capacity. PGV and large event magnitude thresholds should be based on sensor records of previous damaging seismic events.

- Seismic sensor density. As above, the PGV threshold must take into consideration the polygon volume and sensor density. 
- Human comfort factor. It is important to consider the psychological impact of strong ground motion and seismic activity on the workforce, especially in areas of high exposure such as workshops and crusher chambers.

\subsection{Event rate tracking}

The following sections provide a detailed overview of the possible seismic parameters that can be monitored via the STAT system for the defined polygons. The parameters can be broken down into proactive monitoring (event rate tracking) and reactive monitoring (ground motion, event magnitude and seismic system health).

With a rise in seismic activity, there is a recognised increase in the probability that a larger and potentially damaging event may occur. The Short Term Event Rate Tracker was developed by Mendecki (2016a) based on this general concept.

Changes in seismic activity rate within two different time periods, $\Delta t_{1}$ and $\Delta t_{2}$ and within the same volume (polygon) of rock can be achieved by counting the number of accepted seismic events within the respective time period. A potency value threshold, based on the $\mathrm{P}_{\min }$, which represents the potency above which the entire seismic catalogue is captured, for the monitoring polygon is applied to the assessment (Mendecki 2016a). Mendecki also states that "the minimum practical time interval over which the event count is measured and compared against the reference rate is 30 mins; any shorter and the associated uncertainty increases" (Mendecki 2016a). Longer interval windows would also increase the time taken before an elevated activity rate was recognised resulting in unnecessary hazard exposure. Seismic activity rates under normal background conditions can be described as resulting from a Poisson process, and as such, can be irregular in nature. Mendecki states that to measure the seismicity rate change, the probability density function of the activity rate $(\lambda)$ must be derived by normalising the Poissonian density function. As such, the probability that the seismicity rate in two different time intervals increased by more than $\mathrm{k}$ times is:

$$
\operatorname{Pr}\left(\frac{\lambda_{2}}{\lambda_{1}}>k\right)=\frac{1}{N_{2} ! N_{1} !} \int_{0}^{\infty} x^{N_{1}} \exp (-x) \Gamma\left(N_{2}+1, k x \frac{\Delta t_{2}}{\Delta t_{1}}\right) d x
$$

where:

$$
\begin{aligned}
& \lambda_{1}=\mathrm{N}_{1}\left(\geq \log \mathrm{P}_{\min }\right) / \Delta \mathrm{t}_{1} \text { and } \lambda_{2}=\mathrm{N}_{2}\left(\geq \log \mathrm{P}_{\min }\right) / \Delta \mathrm{t}_{2} \text { are the activity rates during } \Delta \mathrm{t}_{1} \text { and } \Delta \mathrm{t}_{2}, \\
& \text { respectively. } \\
& \Gamma\left(\mathrm{N}_{2}+1, \mathrm{kx} \Delta \mathrm{t}_{2} / \Delta \mathrm{t}_{1}\right)=\int_{k x \Delta t_{1} / \Delta t_{2}}^{\infty} \exp (-t) t^{N_{2}+1} d t \text { is the upper incomplete Gamma function. } \\
& x=\lambda \Delta \mathrm{t} .
\end{aligned}
$$

(Marsan \& Wyss 2011; Mendecki 2016a Section 4.2)

Using Equation 2, it is possible to calculate probabilities of activity rate change in real time ( 0.5 hour periods) and display within the STAT application the results using a simple traffic light system, whereby:

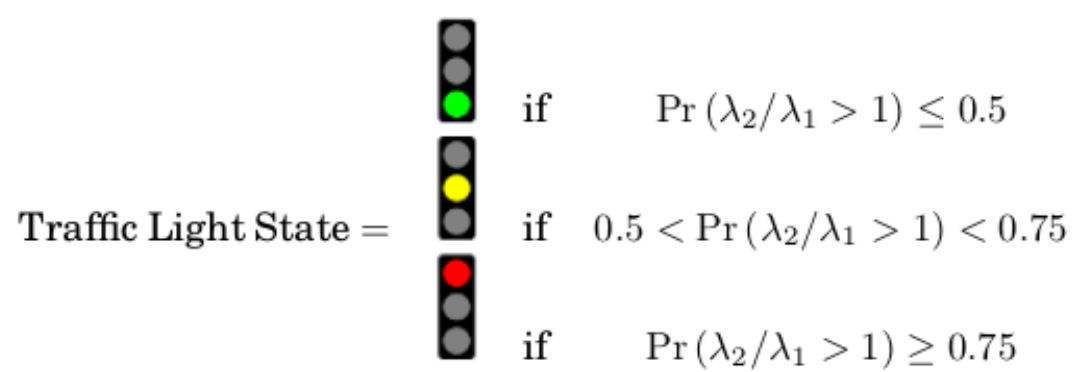


The reference number of events $\left(\mathrm{N}_{1}\right)$ for a given $\mathrm{t}_{1}$ (by default, an hour) is calculated over a background period during which people worked underground, and in which there were no large events and no artificial elevation of seismic activity, e.g. due to blasting. The following criteria should be adopted for setting the reference seismic activity rate:

1. Data recorded during the minimum post-blasting exclusion range is excluded as the seismic response is not assumed to be Poissonian.

2. The time ranges over which data is analysed is aligned with when personnel are working underground (which may be continuous).

3. If a significant event occurred during the chosen time range, then it is excluded as association would increase the estimated background rate.

4. If a particular blast had an unusually high seismic response, then the entire time period up until the next blast is excluded from the calculation. Further, if a large event occurred during good periods (periods when people were underground), then we also exclude all data from the event time to the next blast.

The coefficient of variation is calculated to test that the reference data is close to Poissonian. If the dataset is Poissonian, this value should be close to one.

Once the reference seismic data has been selected from periods when personnel are underground, the periods are divided into $t_{1}$ (usually one hour) bins, as illustrated in Figure 2 . In this example, there are 1,300 observations (hours) where no events occurred within the reference period, about 1,050 hours where only one event occurred, and so on. The correct lambda 1 value is left to the user to choose. However, to avoid excessive triggers from the system, it is recommended to use a higher value than the mean average for the reference rate. To aid in the set-up of the STAT event rate trigger thresholds and other set-up parameters, an automated process or wizard is available that can be initiated manually or automatically.

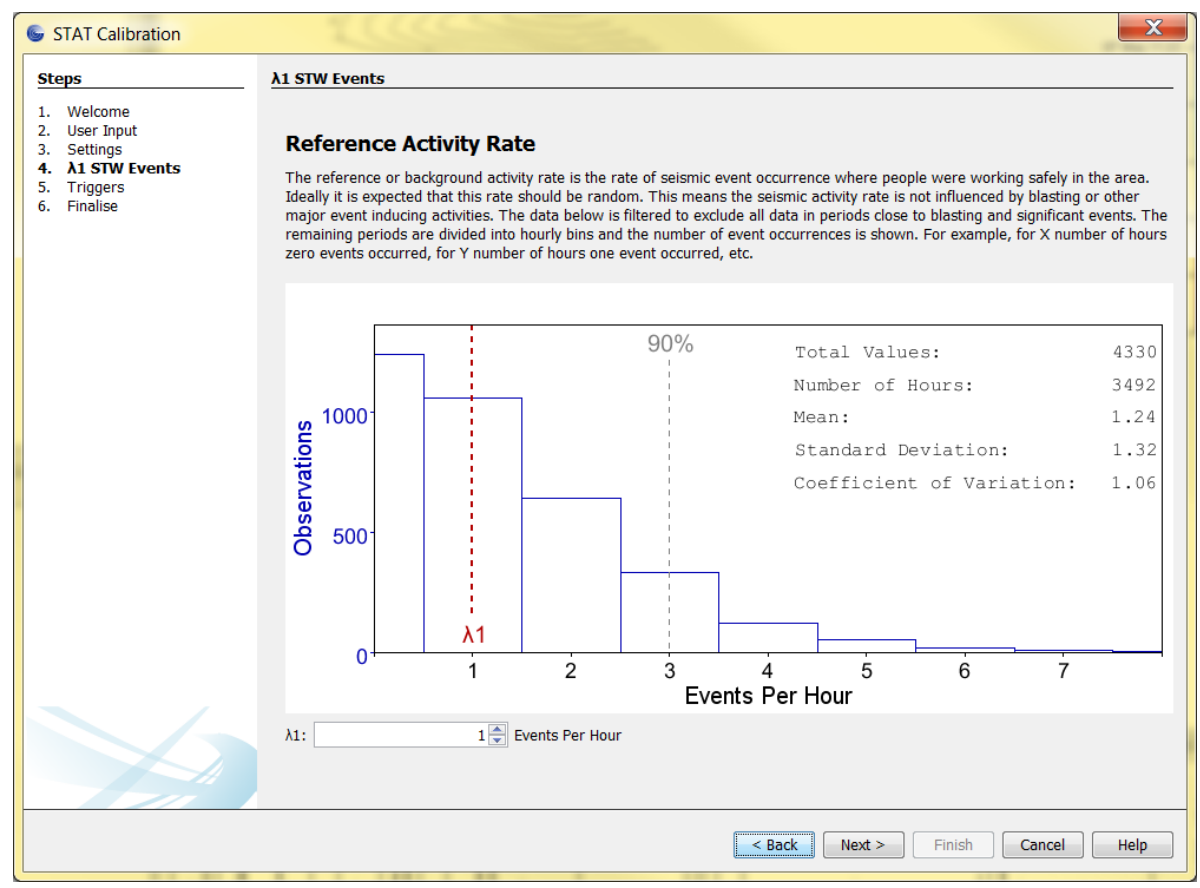

Figure 2 Event rate distribution - STAT calibration wizard

A time line of STAT 'traffic light' status and associated seismic parameters (cumulative potency, cumulative events, event magnitude and distance from polygon centre) for each of the monitoring polygons is shown within the STAT application, an example of which is shown in Figure 3. 


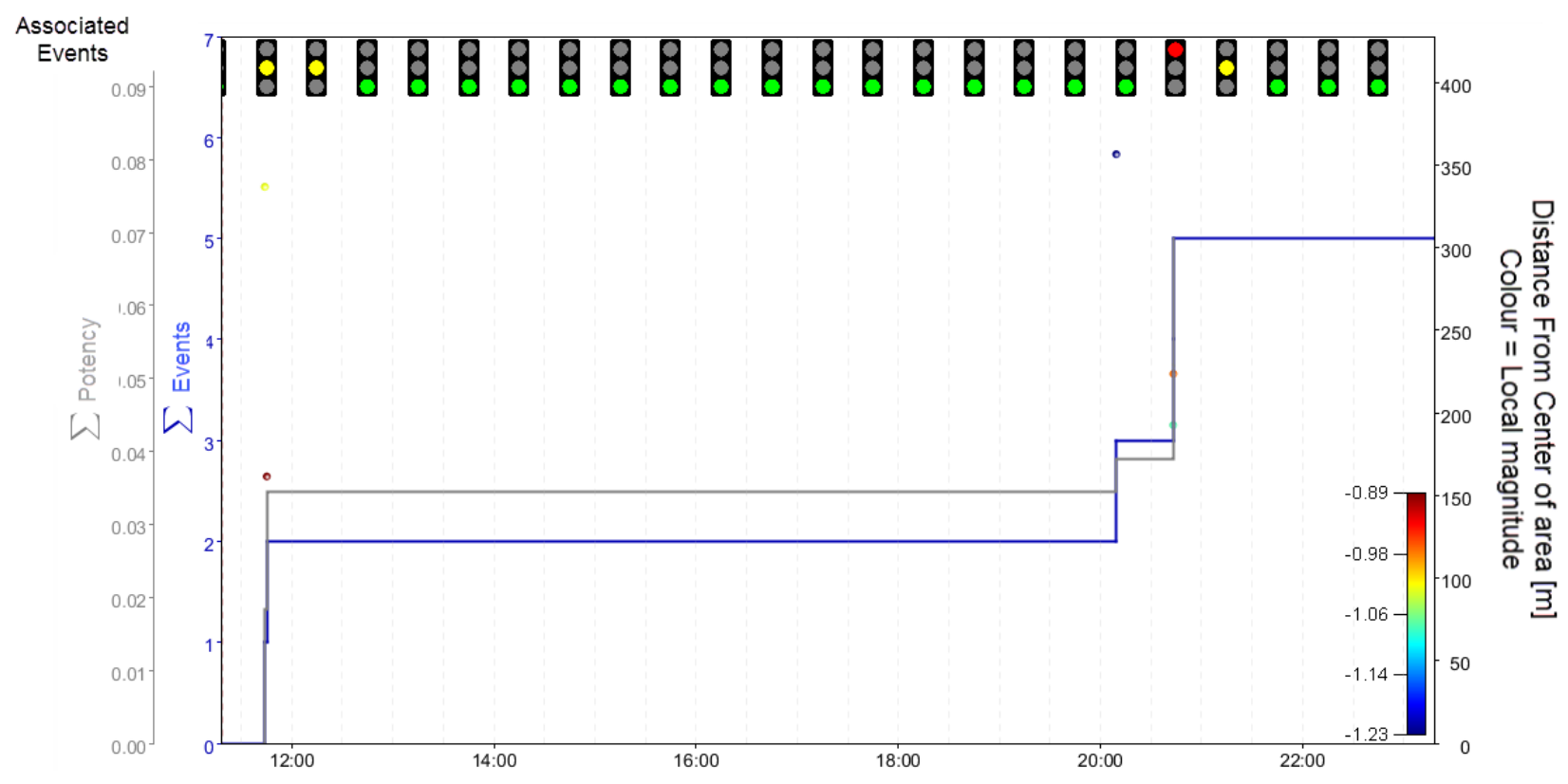

Figure 3 Example of STAT results, illustrating seismic activity rate status change

\subsection{Ground motion}

Seismic alert protocols have traditionally been based on the occurrence of an event above a predefined event magnitude threshold within a monitored area. Good practice would dictate that only manually processed events, i.e. events with an accurate magnitude and minimal location error would be accepted as possible threshold triggers. Although an acceptable approach, there is an inherent delay associated with this method, as processing of an event can take upwards of 10 minutes depending on event processing arrangements. More often than not, the geotechnical engineer is first alerted to the occurrence of large events directly via operations personnel, rather than automated alert systems.

With the desire to have a more immediate alert system for the monitored areas, the STAT application was developed further to include the continuous monitoring of ground velocity, measured at selected seismic sensors (geophones) located within or very close to the monitored polygons. The inclusion of continuous ground velocity monitoring in the STAT application means it is no longer necessary to process a seismic event to generate a reliable trigger, as ground velocity is measured and transmitted immediately with no requirement for processing or manipulation. As such, responses can occur almost immediately when triggered via a breach of ground motion threshold.

The use of ground motion as a trigger requires the monitored area to contain, or have in close proximity, at least two active seismic sensors to avoid false triggers, which can occur due to electrical interference on individual sensors. The minimum number of sensors that must exceed the PGV threshold in order to trigger an alert can be set in the application. This functionality allows the engineer to adjust the trigger sensitivity to reflect sensor spread and monitoring volume. As the monitoring polygon volume increases, the number of active sensors within the volume must also increase; a rule of thumb being one sensor per $10,000-40,000 \mathrm{~m}^{3}$. It is not recommended to rely upon sensors located away from the monitored area, as threshold trigger levels would need to be low in order to take account of seismic attenuation, and as such, are susceptible to false triggers. Increasing threshold levels to counter this risk would subsequently limit the effectiveness of the application.

Some calibration work is required to determine appropriate trigger thresholds, which takes into account recorded PGVs from previous damaging seismic events, the range of possible event hypocentre to sensor distances, and a ground motion prediction equation (GMPE) or equivalent. For initial implementation purposes, the authors suggest utilising the chart in Figure 4 to assist in the determination of appropriate PGV thresholds. The chart integrates the results of a GMPE, developed by Mendecki (2016b) and the rock mass damage scale proposed by Kaiser et al. (1992). In the case shown in Figure 4, the ground support is considered 
to be 'dynamic' in its energy absorption capacity. It should be noted that the results of the GMPE do not take into account the site effect where interaction with excavations can lead to amplification of ground motion felt at the excavation surface. The GMPE is also based on a best fit analysis of seismic sensor data recorded at a cave mining operation, and as such, must undergo systematic calibration.

Within the STAT application, a ground motion threshold breach is distinguished from an activity rate trigger by a changing in colour of the traffic light surround (Figure 5). If the integrated MCTRS application is also being used, as detailed in Section 4, an alert command box will also be activated. The location of the triggered monitoring polygon is also highlighted by a change of colour (Figure 6).

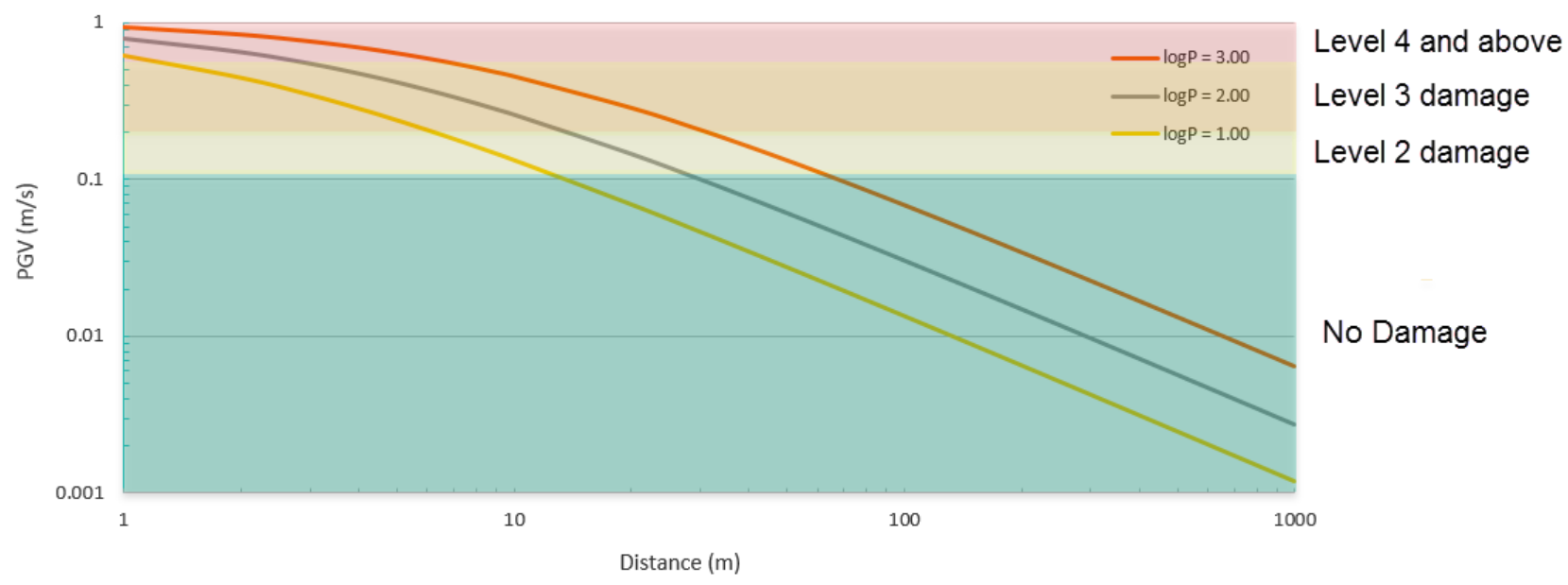

Figure 4 Predicted ground velocity chart (after Mendecki 2016b) with overlain ground support damage forecast (after Kaiser et al. 1992) for dynamic ground support. Note: PGV values do not take into account site effect

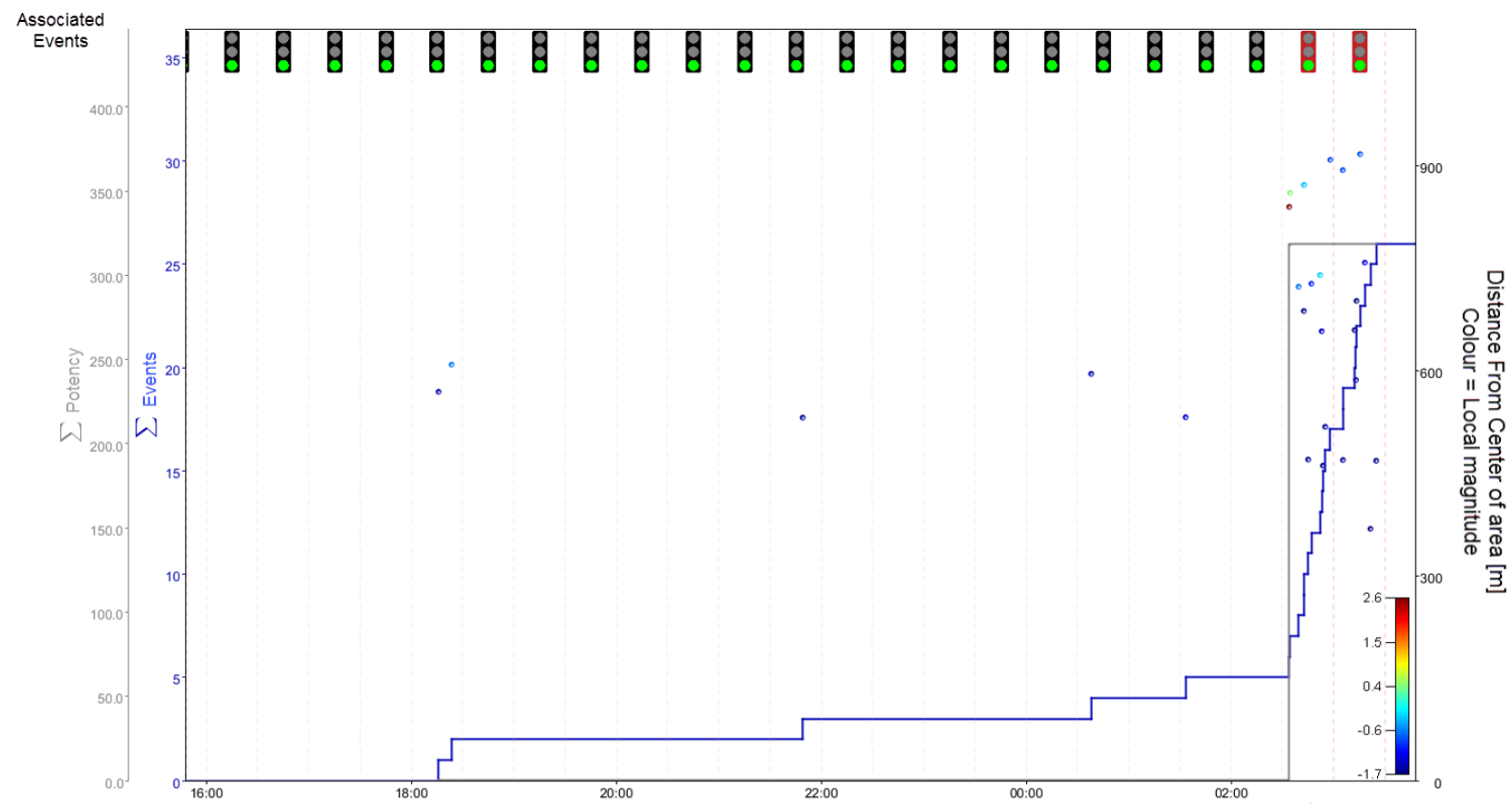

Figure 5 Example of a ground motion or event magnitude trigger alert (red surround) within the STAT application 


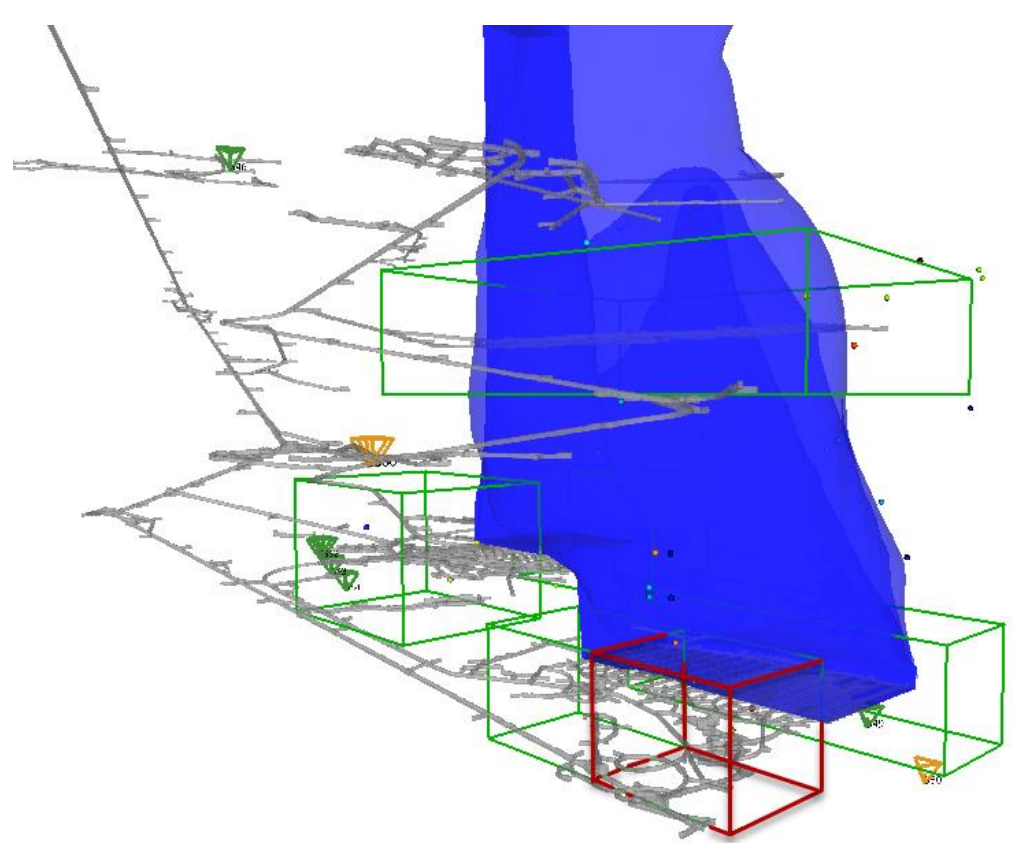

Figure 6 Example of the seismic 3D visualiser with the monitoring polygons coloured by their STAT status

A shortfall of this approach is the course resolution at which the mitigation controls must be placed. While the strong ground motion hazard may be isolated, any mitigation controls would be applied to the entire monitoring polygon. Further development of seismic event ShakeMaps, as described by Meyer et al. (2018) and there integration with the STAT application has the potential to rapidly define the risk-affected areas on a finer resolution, allowing for more focused mitigation control adoption.

\subsection{Event magnitude}

Although it is recommended to use the ground motion parameter to initiate large seismic event mitigation controls, the layout of particular seismic systems may not be set-up to accommodate this. In these circumstances, it is possible to revert to a more conventional event magnitude-based trigger threshold. Thresholds can be tailored for each monitored polygon based on the level of exposure, installed ground support capacity, and previous damaging seismic event records. As mentioned, there is an inherent delay associated with event magnitude triggers, which should be taken into consideration. As with ground motion triggers, to distinguish a magnitude trigger from an activity rate trigger, the alert is visually represented by a change in the background colour of the traffic light (Figure 5). The colour of the activated monitoring polygon volume also changes colour to red to reflect the triggered level (Figure 6).

\subsection{System health}

As seismic monitoring and alert systems become an integral part of a mine's risk management strategy, there is a requirement to monitor the health and performance of the system. The STAT application has been developed to include a real-time system health monitor with predefined threshold triggers, which raises an alert via the MCTRS. A minimum time threshold prevents any false alarms resulting from short, intermittent drops in system health due to breaks in communications or power supply. Through system health monitoring, early intervention can take place to rectify identified issues, or other mitigation controls can be enacted to minimise the workforce's exposure due to reduced monitoring sensitivity or functionality. 


\subsection{Statistical evaluation}

To understand the implication of introducing the STAT application, or adjusting threshold levels, it is possible to run a statistical analysis on the number of threshold triggers. The evaluation can be defined by time period for each of the monitoring polygons. An example of the output can be seen in Table 1. The information can be used to understand and demonstrate the impact of the current or any proposed threshold settings. Long-term changes in the seismic environment of each monitoring polygon may also be highlighted through analysis of threshold trigger statistics.

Table 1 Example of a STAT statistical evaluation

\begin{tabular}{lll}
\hline Rating & Number & Percentage \\
\hline Green & 3,212 & 98.3 \\
Yellow & 50 & 1.5 \\
Red & 4 & 0.1 \\
Red (large events) & 0 & 0 \\
Red (activity) & 4 & 0.1 \\
Red (PGV) & 0 & 0 \\
\hline
\end{tabular}

\section{$4 \quad$ Mine Control Trigger Response System}

The MCTRS integrates with the STAT application to provide a seamless seismic risk management application, from system monitoring to automated alert generation and mitigation control initiation. The MCTRS forms the output interface to the STAT monitoring application and is organised around a tabular seismic TARP. The application is ideal for deployment in mine controls centres, as personnel require minimal seismic knowledge in order to use the application. System operators simply need to enact the predefined controls presented via the alert command box in the event of a threshold breach.

Under normal background seismic conditions, monitored areas (polygons) have their base level TARP category displayed (Figure 7). Any change in condition of the monitored areas will immediately generate a command box (Figure 8), which details the required action associated with the identified seismic condition. An audible alert can also accompany the command box alert. Following confirmation of the command dialog box, which requires the operator to add their name, the required action will also appear in the TARP tab for a predefined period of time. If the alert condition remains, such as high event activity rate, the action will remain in the TARP tab.

\begin{tabular}{|c|c|c|c|c|c|}
\hline Description & Rule & Hazard & Response 1 & Response 2 (Shift Supervisor) & Confirmed \\
\hline & $\begin{array}{l}\text { Crusher- } \\
\text { Workshop - STAT } \\
\text { green light }\end{array}$ & Low & $\begin{array}{l}\text { - - Enter into rock noise register if rock } \\
\text { noise reported } \\
\text { - - Notify on-call Geotech Engineer if } \\
\text { rock noise is reported from }>2 \text { locations }\end{array}$ & $\begin{array}{l}\text { - Follow instructions from on-call Geotech } \\
\text { Engineer regarding exclusion zone / } \\
\text { inspection requirements }\end{array}$ & \\
\hline & $\begin{array}{l}\text { North Abutment } \\
\text { - STAT green light }\end{array}$ & Low & $\begin{array}{l}\text { - - Enter into rock noise register if rock } \\
\text { noise reported } \\
\text { - - Notify on-call Geotech Engineer if } \\
\text { rock noise is reported from }>2 \text { locations }\end{array}$ & $\begin{array}{l}\text { - Follow instructions from on-call Geotech } \\
\text { Engineer regarding exclusion zone / } \\
\text { inspection requirements }\end{array}$ & \\
\hline
\end{tabular}

Figure 7 Example of background seismic TARP category 


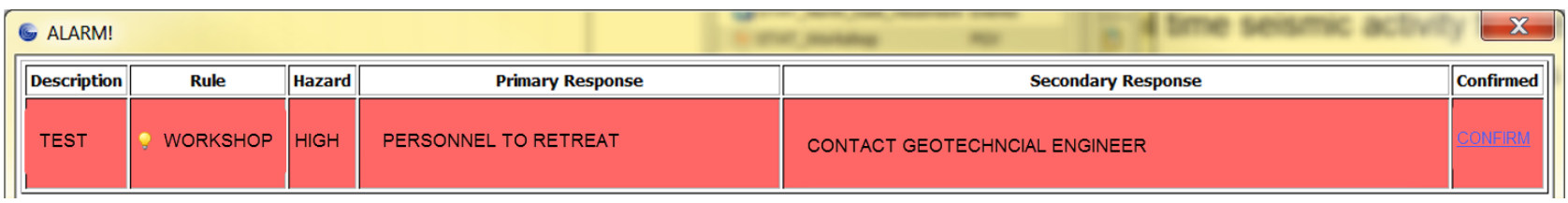

Figure 8 Example of STAT alarm command box

The system creates a log of all alert confirmations, recording time of confirmation, user name (who confirmed), machine name, alert description, and type and kind of alert. This provides a useful time stamped record of alerts and initiation of mitigation controls.

\section{Discussion}

Through the development and implementation of the two applications at two Australian caving operations, the authors have identified the following key benefits.

Reduction in workforce exposure: By removing personnel from areas of increased seismic activity or from areas affected by a seismic event with minimal delay, it is possible to reduce the workforce's exposure, and in turn, reduce the seismic risk. This is considered by the authors to be the major benefit of the applications.

Rapid and automatic response: Through the innovative use of ground velocity measurements, which require no human input, it is possible to rapidly implement mitigation controls within the monitored area with the intention of reducing workforce exposure. With the integrated MCTRS, threshold trigger response actions can be activated immediately through a visual and/or audible alert. This avoids the inherent delays associated with processing a possibly damaging seismic event, interpreting the TARP categories and then communicating the required actions to those who must enact them.

Continuity in response: However detailed and prescribed the TARP categories are, there is still a requirement for interpretation, which can lead to inconsistencies with regards to determining the appropriate category and associated mitigation controls. The integrated STAT and MCTRS applications minimise the need for human interpretation, with clearly defined and agreed thresholds and associated mitigation controls. This not only ensures continuity in interpretation and response, but also reduces human error.

Document version control: As the application is entirely software based, updates to the seismic TARP due to system expansions, lessons learnt, etc. can be implemented rapidly. This eliminates the risks associated with document version control where key stakeholders refer to out-of-date copies of the document.

Reduced susceptibility to spurious triggers: Seismic systems are susceptible to false or spurious events, caused by electrical interference and mechanical noise such as rock breakers. The STAT system reduces this risk via the use of minimum sensor trigger thresholds. This functionality enables the user to set the minimum number of sensors that must achieve the threshold level to trigger an alert.

Monitored area statistical reporting: The seismic database can be queried to determine the number and frequency of triggers within a defined time period for each of the monitored areas, based on the entered threshold settings. This enables the geotechnical engineer to review and demonstrate the impact of the current controls and that of any threshold changes, or the addition of a new monitoring area. Determination of appropriate thresholds for seismic activity rate and predicted ground velocity is an iterative process with threshold levels required at a level that minimises seismic risk exposure and impact on production. Periodic reviews of trigger frequency may also highlight longer term variation in seismic hazard.

\section{Future direction}

The authors are continuing to work with the software developers to implement further improvements to the applications. Further integration of the two applications with set-up wizards will improve the experience of the user and reduce human error when designing and implementing the system. Through the implementation of the ShakeMap application, as detailed by Meyer et al. (2018), it will also be possible to implement more focused 
mitigation controls, rather than the more coarse resolutions approach taken currently, whereby an entire polygon area has mitigation controls applied irrespective of the hazard's location.

Following the successful integration of the two applications, the design of future seismic systems will need to take into account the ground motion trigger application, which will require a more regular distribution of sensors in areas of current and future seismic risk.

\section{Conclusion}

The integrated Short Term Activity Tracker and Mine Control Trigger Response System software application is a relatively simple tool to implement at mining operations, with clear benefits in seismic risk management. Current short-term seismic risk management approaches have recognised shortfalls, which impact on their effectiveness in reducing workforce exposure to seismic risk.

Through the implementation of the applications at two caving operations, improvements have been made to seismic risk management through the reduction in workforce exposure during periods of increased seismic risk. The realised benefits of the integrated applications include real-time monitoring of key seismic parameters and automatic trigger response. The innovative use of measured ground motion as a monitoring input enables an almost immediate trigger response to a threshold breach via the Mine Control Trigger Response System. Furthermore, continuity in seismic data interpretation and triggered mitigation controls are recognised advantages of the applications.

Further development work is proposed to integrate the ShakeMap application, also developed by IMS engineers. Additionally, seismic system designs at the two caving operations will now take into consideration the ground motion application and the requirement to have sensors within areas of concern.

\section{Acknowledgement}

The authors thank the Institute of Mine Seismology and Newcrest Mining Limited for giving permission to publish this work.

\section{References}

Harris, PC \& Wesseloo, J 2015, mXrap, version 5, computer software, Australian Centre for Geomechanics, The University of Western Australia, Perth, https://www.mxrap.com

Heal, DP 2010, Observations and Analysis of Incidence of Rockburst Damage in Underground Mines, PhD thesis, The University of Western Australia, Perth.

Heal, DP, Potvin, Y \& Hudyma, MR 2006, 'Evaluating rockburst damage potential in underground mining', in DP Yale, SC Holtz, C Breeds \& U Ozbay (eds), Proceedings of the 41st US Rock Mechanics Symposium, American Rock Mechanics Association, Alexandria.

Kaiser, PK, Tannant, DD, McCreath, DR \& Jesenak, P 1992, 'Rockburst damage assessment procedure', in PK Kaiser \& DD McCreath (eds), Rock Support in Mining and Underground Construction, A.A. Balkema, Rotterdam, pp. 639-647.

Marsan, D \& Wyss, M 2011, Seismicity Rate Changes, https://dx.doi.org/10.5078/corssa-25837590

Mendecki, AJ 2016a, Mine Seismology Reference Book: Seismic Hazard, 1st edn, Institute of Mine Seismology, Hobart.

Mendecki, AJ 2016b, Development of Ground Motion Prediction Equation for Cadia East Mine, technical report, Institute of Mine Seismology, Hobart.

Meyer, S, Doolan, J, Chester, C \& Basson, G 2018, 'Rapid assessment of the spatial extent of strong ground motion in mines ShakeMap approach', in Y Potvin \& J Jakubec (eds), Proceedings of the Fourth International Symposium on Block and Sublevel Caving, Australian Centre for Geomechanics, Perth, pp. 561-572. 